\title{
CDISC SEND Microscopic Histopathology Result Category Terminology
}

National Cancer Institute

\section{Source}

National Cancer Institute. CDISC SEND Microscopic Histopathology Result Category

Terminology. NCI Thesaurus. Code C90017.

The terminology that includes concepts relevant to the Clinical Data Interchange Standards Consortium (CDISC) Standard for the Exchange of Non-clinical Data (SEND) microscopic histopathology result category. 